\title{
"The role as a champion is to not only monitor but to speak out and to educate": the contradictory roles of hand hygiene champions
}

Cassie Cunningham Goedken ${ }^{1 *}$ (D), Daniel J. Livorsi ${ }^{1,2}$, Michael Sauder $^{3}$, Mark W. Vander Weg ${ }^{1,2,4}$, Emily E. Chasco ${ }^{1}$, Nai-Chung Chang ${ }^{5}$, Eli Perencevich ${ }^{1,2}$ and Heather Schacht Reisinger ${ }^{1,2}$

\begin{abstract}
Background: Implementation science experts define champions as "supporting, marketing, and driving through an implementation, overcoming indifference or resistance that the intervention may provoke in an organization." Many hospitals use designated clinical champions-often called "hand hygiene $(\mathrm{HH})$ champions" - typically to improve hand hygiene compliance. We conducted an ethnographic examination of how infection control teams in the Veterans Health Administration (VHA) use the term "HH champion" and how they define the role.

Methods: An ethnographic study was conducted with infection control teams and frontline staff directly involved with hand hygiene across 10 geographically dispersed VHA facilities in the USA. Individual and group semistructured interviews were conducted with hospital epidemiologists, infection preventionists, multi-drug-resistant organism (MDRO) program coordinators, and quality improvement specialists and frontline staff from June 2014 to September 2017. The team coded the transcripts using thematic content analysis content based on a codebook composed of inductive and deductive themes.

Results: A total of 173 healthcare workers participated in interviews from the $10 \mathrm{VHA}$ facilities. All hand hygiene programs at each facility used the term $\mathrm{HH}$ champion to define a core element of their hand hygiene programs. While most described the role of $\mathrm{HH}$ champions as providing peer-to-peer coaching, delivering formal and informal education, and promoting hand hygiene, a majority also included hand hygiene surveillance. This conflation of implementation strategies led to contradictory responsibilities for $\mathrm{HH}$ champions. Participants described additional barriers to the role of $\mathrm{HH}$ champions, including competing priorities, staffing hierarchies, and turnover in the role.

Conclusions: Healthcare systems should consider narrowly defining the role of the $\mathrm{HH}$ champion as a dedicated individual whose mission is to overcome resistance and improve hand hygiene compliance-and differentiate it from the role of a "compliance auditor." Returning to the traditional application of the implementation strategy may lead to overall improvements in hand hygiene and reduction of the transmission of healthcare-acquired infections.
\end{abstract}

Keywords: Hand hygiene, Champion, Implementation science, Implementation strategy, Infection prevention

\footnotetext{
* Correspondence: cassie.goedken@va.gov

${ }^{1}$ Center for Access \& Delivery Research and Evaluation, lowa City VA Health

Care System, 601 Hwy 6 West, lowa City 52246, USA

Full list of author information is available at the end of the article
}

(c) The Author(s). 2019 Open Access This article is distributed under the terms of the Creative Commons Attribution 4.0 International License (http://creativecommons.org/licenses/by/4.0/), which permits unrestricted use, distribution, and reproduction in any medium, provided you give appropriate credit to the original author(s) and the source, provide a link to the Creative Commons license, and indicate if changes were made. The Creative Commons Public Domain Dedication waiver (http://creativecommons.org/publicdomain/zero/1.0/) applies to the data made available in this article, unless otherwise stated. 


\section{Background}

Hand hygiene is widely considered the most effective method of preventing healthcare-acquired infections (HAIs) $[1,2]$. However, hand hygiene compliance remains persistently low [1-4]. In the USA, the Joint Commission's 2019 National Patient Safety Goal challenges hospitals to lower the risk of HAI by utilizing current Centers for Disease Control and Prevention [5] or World Health Organization hand hygiene guidelines $[2,6]$. In addition, it recommends that hospitals set their own goals for hand hygiene compliance and work toward improving compliance toward those goals [7]. One approach many hospitals use to improve hand hygiene compliance is the recruitment of clinical champions-often called "hand hygiene (HH) champions."

It is well accepted that champions are important in implementation and quality improvement projects [8]. Champions have been defined within the field of implementation science as "individual(s) who dedicate themselves to supporting, marketing, and driving through an implementation, overcoming indifference or resistance that the intervention may provoke in an organization" ([9], p. 9). The concept of a champion to influence change has existed for several decades [10-12]. Although the definition has evolved, many key aspects remain the same; namely, champions are dedicated individuals who attempt to influence and elicit change. Champions are often referred to by a variety of different titles, such as opinion leaders, sponsors, internal entrepreneurs, and change agents [13, 14].

Versions of champions have also been used successfully to improve hand hygiene compliance. Hand washing champions were identified as playing a key role in improving physician hand hygiene compliance at a large, urban hospital in the Midwestern USA [15]. A new resident-trained hand washing champion was identified each day. They provided immediate feedback to inpatient general pediatric teams regarding hand hygiene performance and verbal reminders that were associated with a sustained increase in hand hygiene compliance [15]. Similarly, Saint and colleagues [16] increased healthcare worker ( $\mathrm{HCW})$ hand hygiene compliance across five units in Tuscany, Italy, with a multimodal intervention that included identifying champions on each hospital unit through the presence of green buttons on their clothing promoting hand hygiene, as well as modeling proper hand hygiene behavior. Further, Patel et al. [17] reported hand hygiene compliance improvements before and after patient contact by employing hand hygiene champions to facilitate a variety of intervention activities, including HCW education and providing feedback to units based on audits conducted by an independent infection control team at a hospital in Cape Town, South Africa.
Despite the success of champions used in specific interventions, little is known about whether and how healthcare systems have employed the implementation strategy of clinical champions to promote hand hygiene. As the field of implementation science matures, it is critical to examine how implementation strategies are being used and defined in real-world settings. While it is difficult to find a single, well-accepted definition of ethnography $[18,19]$, most would agree "its emphasis is on the description and analysis of "the everyday" - routine behaviors in their natural settings ([20], p.326)." The goal of this study was to conduct ethnographic research of hand hygiene programs at 10 Veterans Health Administration (VHA) hospitals to understand key components and "everyday" practices of the programs prior to conducting a cluster-randomized control trial of specific hand hygiene interventions [21]. Ethnographic examination of program components as they are discussed and used by healthcare workers illuminates the gaps between conceptualizations defined by experts and real-world applications. One of the key practices identified at each of the facilities was hand hygiene champions. In this paper, we examine the real-world use of the term "champion," including whether the term was used in these programs, how the term was defined, and what the role of $\mathrm{HH}$ champions entailed when used to promote hand hygiene compliance. Conducting this research allowed us to compare the use of the term among the participating Veterans Affairs (VA) hospitals with its application in the literature.

\section{Methods}

As part of a multicenter cluster-randomized trial testing three hand hygiene improvement interventions, an ethnographic study was conducted with infection control teams and frontline staff directly involved with hand hygiene across 10 geographically dispersed Department of Veterans Affairs Healthcare Systems (VAHCSs) in the USA. We conducted site visits at six facilities, which included observations of infection control practices, semistructured interviews with staff directly involved with hand hygiene (hospital epidemiologists, infection preventionists, multi-drug-resistant organism (MDRO) program coordinators, and quality and patient safety staff), and focus groups with frontline staff (two different units, one day shift and one night shift). Staff directly involved with hand hygiene participated in semi-structured phone interviews at four additional VAHCS facilities (Fig. 1). Data were collected at two different time points. The first round of data collection (Time 1 [T1]) occurred between June 2014 and March 2015, and the second round (Time 2 [T2]) occurred between January 2017 and September 2017. T1 data collection was conducted prior to implementing hand hygiene improvement interventions 


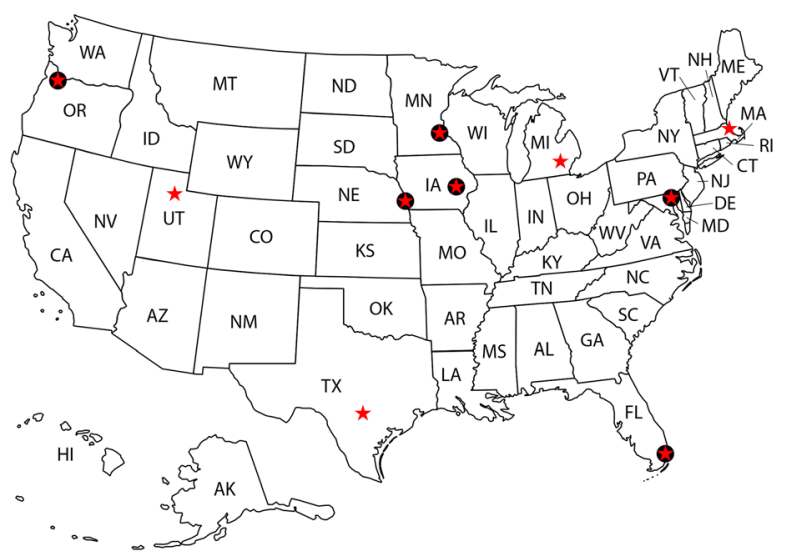

Site Observations
Baltimore, MD
lowa City, IA
Miami, FL
Minneapolis, MN
Omaha, NE
Portland, OR
$\star$ Phone Interviews Only
Ann Arbor, MI
Boston, MA
Salt Lake City, UT
San Antonio, TX

Fig. 1 Qualitative evaluation

for the cluster-randomized trial. For T2 data collection, we conducted site visits at four of the original six hospitals since two hospitals were unable to implement the study interventions due to lack of personnel.

An ethnographic approach was chosen for both methodological and epistemological reasons [22]. From the breath of methods within the ethnographic methodology $[23,24]$, we chose to conduct semi-structured individual and group interviews and observations at six sites and supplement the data with phone interviews from an additional four sites. We also collected organizational documents (e.g., hand hygiene protocols, data collection forms) from all 10 sites. For this paper, we focus on the semi-structured interview data due to the emphasis on how they defined "hand hygiene champions" and the need to focus on the in vivo language used by the participants. We also report on our review of organizational documents and observations to provide context on the organizational structure of the hospitals' hand hygiene programs.

The semi-structured interview guides were developed by the interdisciplinary research team who had substantial expertise in hospital epidemiology and infection prevention. The interview guides were iteratively revised as new data was collected and based on feedback from the participants. Interview guide questions included questions to elicit past and current strategies used for promoting hand hygiene compliance, descriptions of hand hygiene surveillance practices, and how roles and responsibilities related to hand hygiene were structured at the facility level. The focus group guide was developed in the same way and included questions about frontline staff's (e.g., nurses, physicians, unit clerks, and environmental service workers) knowledge and perceptions of strategies to promote and monitor hand hygiene compliance on their unit and in their facility more broadly. We also collected data on hospital staff perceptions of the three bundled interventions, but those data were not included in this analysis.

Interviews were audio recorded and transcribed by trained transcribers. Transcriptions were reviewed against original recordings by the interviewers. All transcripts were imported into MAXQDA qualitative data management software [25]. The study was approved by the VA Central Institutional Review Board and Research and Development Committee at the Iowa City VAHCS, and informed consent was reviewed with all participants.

\section{Analysis}

We conducted a thematic content analysis [26, 27] with an interdisciplinary team that included ethnographically trained social scientists from anthropology and sociology, a health psychologist with experience in infection prevention, and a hospital epidemiologist. Data from T1 of the collection were analyzed across four phases. First, three transcripts were reviewed by the analysis team, and a codebook was developed based upon a priori research questions and emergent content [28]. During the first phase, the transcripts were coded using parallel processes. Forty-nine percent of transcripts were coded by consensus at biweekly meetings among the analysis team. All coders participated in the biweekly meetings. Two coders from the analysis team coded the remaining transcripts (51.2\%) during this same period. Each coder first coded independently and then met to reach consensus and enter the coding in MAXQDA. This process of continuous dialogue increases the validity and reliability of the coding process by refining the content boundaries of codes and improving coding consistency.

In the second phase of analysis with $\mathrm{T} 1$ data, we subcoded the two most frequently applied codes "hand hygiene intervention strategies" and "hand hygiene monitoring." 
"HH champion" was a subcode that cut across both highfrequency codes. We applied the subcode to segments of text in which "HH champion" was used verbatim (in vivo). We also noted segments of text that were conceptually similar, but in which the term "HH champion" was not used. Conceptually, "HH champion" included references to individuals responsible for surveillance, peer-to-peer coaching, formal and informal education, and general promotion of hand hygiene on a unit or within their own clinical group. We subcoded all text segments under these conceptually related categories as "HH champion."

In phase three of the analysis, we used the hierarchical codebook (codes and subcodes) developed with T1 data to code all transcripts from the T2. Thirty-five percent of transcripts were coded via group consensus, and 65\% were coded by at least two coders.

In the final phase, we tested the "HH champion" subcode by conducting a lexical search of all occurrences of "champion" (or champions) in the transcripts and compared the lexical search to all "HH champion"coded segments.

\section{Results}

A total of 38 individuals responsible for or involved with hand hygiene programs participated in semi-structured interviews, while 70 frontline staff participated in focus groups in the T1 interviews. In T2 interviews, 32 individuals responsible for or involved with hand hygiene programs participated in semi-structured interviews, and 33 frontline staff participated in focus groups.

Descriptive characteristics of staff are included in Table 1 (T1) and Table 2 (T2). Infection preventionists (T1, 14\%; T2, 19\%) composed the largest proportion of staff, with the remainder divided among hospital epidemiologists (T1, 9\%; T2, 12\%), MDRO program coordinators (T1, 7\%; T2, 6\%), and others (e.g., quality and patient safety) (T1, 7\%; T2, 12\%). The largest proportion of the frontline staff sample was composed of nurses (T1, 64\%; T2, 46\%).

\section{The concept of "HH champions"}

Participants often referred to the concept of a "HH champion" to describe a range of roles, including monitoring hand hygiene compliance, informal and formal HCW education, and general promotion of hand hygiene. Of the 233 "HH champion"-coded segments, the lexical search showed $49.8 \%(n=116)$ of participants specifically use the term "champion" and in all but one interview the participant used the term champion before the interviewer (i.e., without prompting by the interviewer). In our analysis, we found all 10 sites used both the concept of $\mathrm{HH}$ champions and the specific term.
Table 1 First round data collection (T1) participant characteristics at $10 \mathrm{VAHCs}$ hospitals

\begin{tabular}{ll}
\hline Individuals responsible for $\mathrm{HH}$ characteristic & Total participants $(\mathrm{N}=38)$ \\
Mean age (SD) & $49.91(9.23)$ \\
Mean tears at VA (SD) & $13.85(9.96)$ \\
Occupation & 10 \\
Hospital epidemiologist & 15 \\
Infection preventionist & 7 \\
MDRO PC & 6 \\
Others (e.g., quality, patient safety) & Total participants ( $N=70)$ \\
Frontline staff characteristic & $41.66(11.2)$ \\
Mean age (SD) & $8.19(9.07)$ \\
Mean years at VA (SD) & \\
Occupation & 53 \\
Nursing & 3 \\
Medial & 3 \\
EMS & 3 \\
Admin & 7 \\
Other & 3 \\
\hline MDO PC, multi-drug-resistant organism prevention program coordinator
\end{tabular}

$M D R O P C$, multi-drug-resistant organism prevention program coordinator

\section{Organizational structure of hand hygiene programs}

Based on analysis of the organizational documents $(n=$ 10 sites) and observational field notes ( $n=6$ sites) and supplemented by interviews with hand hygiene program staff $(n=38)$, the organizational structure of hand hygiene programs varied across all 10 hospitals. Hand hygiene was managed by Infection Control at six hospitals, whereas in the remaining four facilities it fell under the umbrella of Quality and Patient Safety. Despite the

Table 2 Second round data collection (T2) participant characteristics at 8 VAHCS hospitals

\begin{tabular}{ll}
\hline Individuals responsible for HH characteristic & Total participants $(N=32)$ \\
Mean age (SD) & $47.31(9.84)$ \\
Mean years at VA (SD) & $11.93(9.31)$ \\
Occupation & \\
Hospital epidemiologist & 12 \\
Infection preventionist & 4 \\
MDRO PC & 8 \\
Others (e.g., quality, patient safety) & Total participants $(N=33)$ \\
Frontline staff characteristic & $38.12(11.84)$ \\
Mean age (SD) & $5.08(6.00)$ \\
Mean years at VA (SD) & \\
Occupation & 30 \\
Nursing & 2 \\
EMS & 1 \\
Other &
\end{tabular}

$M D R O P C$, multi-drug-resistant organism prevention program coordinator 
difference in oversight, infection control teams at all 10 sites were responsible for reporting hand hygiene compliance rates to hospital leadership. Three of the six observed sites displayed hand hygiene compliance data on at least one unit of the hospital. All hand hygiene programs reported using HCWs to monitor the hand hygiene compliance of their fellow HCWs on their units. This is similar to findings from the 2012 survey of VHA facilities in which we found $98.6 \%$ of facilities used direct observation to monitor hand hygiene [29]. In addition to monitoring hand hygiene compliance, some were also responsible for entering observations into an electronic system for reporting. Many described this role as " $\mathrm{HH}$ champion"; however, the responsibilities of $\mathrm{HH}$ champions varied and often included providing peer-to-peer coaching, delivering informal education, and general promotion of hand hygiene, in addition to their surveillance role. Below, we present the analysis of the elements that emerged from the ethnographic data to define the role of $\mathrm{HH}$ champions as it played out in realworld clinical practice.

\section{HH champion: surveillance}

The subtheme "surveillance" focused on the responsibility of collecting hand hygiene compliance data. Within this subtheme, participants used terms such as surveillance, monitoring, and observation and discussed whether or not the collection of compliance data was covert.

Many sites reported utilizing $\mathrm{HH}$ champions to conduct monitoring of hand hygiene compliance. This surveillance typically came from an individual chosen by the unit's nurse manager, although sometimes the individual would volunteer for the role:

... usually each area will identify a person to, you know, be a--, do their hand hygiene monitors and/or delegate the hand hygiene monitors, or just kinda be the champion for that area. [program analyst, site 6, T2]

Some hospitals had covert observers, while other facilities wanted the observers to be known and able to provide immediate feedback. As one staff member in charge of a facility's hand hygiene program noted:

We used to call them secret shoppers. I prefer to call them hand hygiene champions, like people that are championing, that are collecting observations. Some people know who they are, some people don't. They just know that there's somebody watching them in their area, which I think is, is always good when you know people are out there watching you. [frontline staff, site 8, T2]

Others intentionally chose not to have champions conduct observations covertly:
We went out and we did teaching and the observers, they were no longer secret. They were observers and they were proactive, going to the person and let them know--, (I was) observing hand hygiene and, "I noticed that you came out. We would like you to have your hands wash [ed] or use hand sanitizer in order to prevent an (infection)." [quality/patient safety lead, site 9, T1]

In these examples, surveillance is a part of the $\mathrm{HH}$ champion role, but informal education or coaching was also part of the responsibility at some sites.

\section{$\mathrm{HH}$ champion: formal and informal education}

The subtheme "formal and informal education" refers to another responsibility held by the $\mathrm{HH}$ champions. Specifically, this theme describes the different styles/ways in which $\mathrm{HH}$ champions must educate fellow HCWs on hand hygiene practices, which may include encouragement/coaching, reminders, and informing/teaching. Although participants indicated that hand hygiene education was typically done both formally and informally, $\mathrm{HH}$ champions were generally tasked with informal education and peer-to-peer coaching.

\section{We (the infection control team) always encourage them [HH champions] to speak up. So if it is one of like our nurse observers, we would-, if they want to speak up, we don't want them to come out themselves as somebody that may be doing observations, but if they could (... ) try to encourage each other to do the right thing. [infection preventionist, site 8, T2]}

Having staff on the unit champion hand hygiene was considered desirable from an infection control standpoint. The belief was as follows: If staff are involved in hand hygiene by reminding and educating one another, this may help shift the culture on the unit and improve hand hygiene compliance by raising staff awareness.

This (being the HH champion on a unit) is a constant reminder, the fact that they (HH champion) are doing this (...) it's a reminder for them (HH champion) to participate in hand hygiene and to be well aware of people who don't, you know. [infection preventionist, site 9, T1]

(A HH champion is) somebody who works in the area, who people know, who can really, you know, engage people to change their practice. [program analyst, site 6, T2]

One nurse elaborated on this idea of culture change and explained how the observers on the units provided education to staff through direct feedback, which she 
believed could lead to making hand hygiene compliance routine.

When we have people as champions they can change the culture of the floor. We definitely encourage them to say something. I'm constantly saying, "This is your floor; you protect that patient and you know if you see something not right you tell someone. You tell them." You know this is a part of their role as a champion is to not only monitor but to speak out and to educate." [infection preventionist, site 5, T1]

\section{HH champion: barriers}

"Barriers" is a subtheme we used to capture the many challenges associated with being the $\mathrm{HH}$ champion. Challenges ranged from difficulties navigating the hospital hierarchy to sufficient time for their $\mathrm{HH}$ champion responsibilities. We found unique barriers associated with the roles $\mathrm{HH}$ champions held. These barriers existed when $\mathrm{HH}$ champions were solely acting in the auditing or surveillance role, but they also proved to be a challenge when $\mathrm{HH}$ champions were tasked with the combined roles of auditors and educators.

As noted, having staff monitor hand hygiene on their own unit has its advantages, such as being able to provide education and immediate feedback to peers-"their role as a champion is to not only monitor but to speak out and to educate." [infection preventionist, site 5, T1]. However, this combined role also has its disadvantages. These disadvantages reflect the contradictory roles of the $\mathrm{HH}$ champion: the responsibility to accurately monitor and report the behavior on the unit for purposes of quality improvement and the responsibility to promote hand hygiene and educate peers.

\section{I: Okay. Um, are they secret champions or-?}

3113: Um, some are and some aren't. So it's all with preference. I DO like to tell them to be known as a hand hygiene champion for their unit or their area. I try to tell them this, "If you're observing someone and they're consistently non-compliant and you don't say anything, then yeah, you're getting decent data, but you're not-, you're not acting on the cause." We're trying to prevent infections by increasing awareness, so I do try to push that they be known to their unit as a champion, if you're around, people will do hand hygiene. [infection preventionist, site 5, T2]

This same infection preventionist went on to talk about the challenges regarding the responsibilities of hand hygiene compliance in the context of staff hierarchies, which is a recurring theme among the barriers described below:

"This is your patient. You are responsible for this patient. You're, you're responsible in preventing infections, and if you're doing hand hygiene and then, say, a physician walks in and doesn't do hand hygiene, well, you know, there goes all your effort."

In addition, staff who are observing their peers report feeling conflicted about their dual role as colleague/peer observer. They do not want to get their fellow HCWs or their unit in trouble, and therefore may report only the "good" observations they observe.

We basically have champions in all our units, they do hand hygiene observations. (... ) The disadvantage of our program is that in some ways you have the fox guarding the hen house so (... ) you may have more positive responses than really are." [hospital epidemiologist, site 5, T1]

Further, through interviews with frontline staff, we found some peers request special treatment if they know who in the unit is collecting the observations.

(... ) they'll (frontline staff) step away and be like, "don't write me up," out there you know you (HH champion) give people chances [frontline staff, site 9, T2]

HH champions whose primary role was to provide education and promote good hand hygiene often discussed the difficulties of navigating boundaries and dealing with the hierarchy within their facilities.

I think people who are afraid to say something to someone who is maybe higher ranking, and remind them although we keep saying it's okay to remind people, it's important that you do. I think the nurses are--, after a while they get tired of you know telling the next crop of residents coming in, you know, surgical residents that don't wash their hands, don't put on gloves when they handle dressings, you know, after years and years of that, they kind of wear out I think. [infection preventionist, site 3, T1]

One nurse in charge of hand hygiene at her facility explained how staff often defer this responsibility onto the infection control team.

Hand Hygiene Nurse: When staff have concerns with a physician not practicing, they call [name of infection preventionist]. 
Interviewer: Is there any reason why?

Hand Hygiene Nurse: I think just because she's higher up the chain of hierarchy and staff nurses don't always feel comfortable challenging a doc about their hand hygiene or infection control practices. [infection control nurse, site 1, T2]

Time and sustainability are common barriers cited by $\mathrm{HH}$ champions who serve in the hand hygiene auditing and education roles. We found insufficient time to be a disadvantage of tasking staff on the unit as $\mathrm{HH}$ champions. Frontline staff's primary job is patient care. Therefore, they may be too busy to fulfill the role of the $\mathrm{HH}$ champion, adequately monitor hand hygiene compliance, and provide hand hygiene education, as well as coach other HCWs about proper hand hygiene compliance.

I mean we're asking nursing personnel, you know, to give up whatever they give up. I don't know if they give up anything but, you know, they're in charge of doing patient care and we're asking them to do hand hygiene. And I know it creates a problem for some of them." [MDRO program coordinator, site 10, T1]

In addition, sustainability is commonly cited as a barrier to having floor staff champion hand hygiene. $\mathrm{HH}$ champion turnover, staff shortages, and staff reassignment to different units to accommodate the ebb and flow of the patient census contribute to this. Further, when staff become too busy to be the champion, it becomes a rotating role, which can reduce continuity and diffuse responsibility.

\section{Counter example: division of responsibilities}

Although all sites used the term $\mathrm{HH}$ champion, the responsibilities assigned to the role were not uniform. Of the ten sites, two specifically attempted to maintain the anonymity of hand hygiene observers, although they still called the role $\mathrm{HH}$ champions. Other sites took advantage of opportunities such as using summer student interns to audit the HH champions' observations, but these roles were not labeled as champions. However, barriers exist in this model as well.

... one of the work areas here, the person that was the champion in that work area was actually identified by the manager in the area to just briefly discuss their experience with the process and the person actually came up here (infection prevention team office space) and said, "I feel kind of-, I've been outed by the manager. How successful am I going to be anymore?" [infection preventionist, site 4, T1]
In short, this $\mathrm{HH}$ champion felt being "outed" and losing anonymity diminished her ability to succeed in monitoring her peers. At the same time, she could not educate her peers and promote hand hygiene on their unit, while remaining an anonymous $\mathrm{HH}$ champion.

In addition, some infection control teams whose $\mathrm{HH}$ champions had dual responsibilities suggested it would be more effective to separate the educational component from surveillance.

And what we needed were people that didn't work on the units, so we needed respiratory therapists. We needed EMS (Environmental Management Service). They were great. People passing trays, the nursing supervisors (... ) you're always expected to be everywhere all the time. Those people are your secret observers. 'Cause nobody says to them, "What are you doin' here?" You know? And then if we had light duty people, we would give them a good cover story of like why they were there, what they were doing, who they were helping. AND if anybody's cover got blown, they were immediately removed from being an observer. So that's how we got TRULY secret observations.

[infection preventionist, site 9, T2]

\section{Discussion}

$\mathrm{HH}$ champions are used as an implementation strategy across many hospitals in the VHA to help improve hand hygiene compliance. Our ethnographic study examined how HH champions are being employed in "everyday" healthcare settings [20]. Our findings indicate many hand hygiene programs have merged at least two different implementation strategies into the role of the $\mathrm{HH}$ champion: (1) audit and feedback (hand hygiene surveillance) and (2) champions (promotion, coaching, and education). Analyses indicated that this description of the $\mathrm{HH}$ champion role tends to be contradictory. While frontline staff are accessible on the units to conduct surveillance and realtime coaching when they see non-compliant HCWs, their surveillance data are considered inaccurate because HCWs are more compliant when they see the HH champion. Participants both explicitly and implicitly referenced the Hawthorne effect in these instances [30]. In addition, we found the $\mathrm{HH}$ champions often feel pressure to capture and report the "good" hand hygiene of their peers on their unit. Barriers also include having to balance patient care and $\mathrm{HH}$ champion responsibilities at their facility. Other studies have demonstrated similar findings in which facilities struggled to implement clinical champions where different hierarchical levels did not have functional relationships [31-34]. On the other hand, we found important benefits to using $\mathrm{HH}$ champions (in the standard definition). Frontline staff in $\mathrm{HH}$ champion roles can help 
change the culture on their floor to improve hand hygiene compliance through the promotion of hand hygiene, informal coaching, and reminding their peers of proper hand hygiene behavior. In addition, although the accuracy of data is often questioned, $\mathrm{HH}$ champions as described by participants in this study supply an important patient safety metric.

Influencing change in providers' behavior is difficult. Evidence suggests we need to "identify and prepare champions" to support the interventions or practice being implemented [9]. However, it is difficult to understand the role of champions, since a variety of terms have been used in healthcare-related implementation literature [35]. While the terms are different- "change agent" and "opinion leader"-they typically refer to the construct of the "champion" [35]. Miech and colleagues [35] identified over 26 characteristics of effective champions ranging from enthusiasm and energy to drive the implementation process to strong educator and presentation skills to having political acumen. With all of these skills making an effective champion, it is not without reason that applications of the implementation strategy in real-world healthcare settings have expanded to include additional skills and responsibilities such as audit and feedback. In addition, we conducted an ethnographic study to examine the real-world implementation of hand hygiene programs and found all 10 geographically dispersed sites used the term $\mathrm{HH}$ champion to describe a core component of their hand hygiene program. For this reason, we compared their utilization of the term to that used by implementation experts [9]. However, the barriers we found associated with using champions as an implementation strategy are similar to those found in recent work in the area of facilitation as an implementation strategy $[36,37]$. The work in the area of facilitation points to a need to assess the context for the best-matched type of facilitation, ranging from task or goal-oriented to holistic, emancipatory approaches [38]. With all ten sites using the term champion in a similar way, it suggests little assessment and tailoring of implementation strategies to fit the context.

Although we focused on the term champions, surveillance was described as a key component of the $\mathrm{HH}$ champion's role. In the taxonomy of implementation strategies, surveillance falls under audit and feedback, which has been defined as, "collect and summarize clinical performance data over a specified time period and give it to clinicians and administrators to monitor, evaluate, and modify provider behavior" [9, p. 8]. Audit and feedback have been used for decades to influence provider behavior. Research suggests that providing feedback to clinicians about their behavior on a recurring basis leads to important improvements in performance [39]. However, in a systematic review specific to hand hygiene, Gould and colleagues [4] found it difficult to draw conclusions on whether or not audit and feedback interventions could be sustained, largely due to the Hawthorne effect influence on performance behavior. Our own research building off of the same ethnographic study points to multiple barriers to the use of audit and feedback as a strategy for hand hygiene compliance, particularly when using direct observation as the surveillance method [40]. On the other hand, studies have also shown success with designated HCWs providing immediate hand hygiene feedback [15-17]. Further, Patel and colleagues (2016) echoed some of the same barriers in their study of $\mathrm{HH}$ champions, including time constraints to performing their daily responsibilities and champion duties, staff shortages, and turnover of the HH champion [17]. Patel and colleagues' study use of $\mathrm{HH}$ champions was within the definitional boundaries described by implementation scientists $[9,34]$. We are aware of no other hand hygiene compliance study that has used champion in this way. At the same time, many research studies have used audit and feedback, conducted both covertly and openly, as an implementation strategy to improve hand hygiene. Schweizer et al. [41] completed a systematic review and meta-analysis of hand hygiene improvement trials. Combined results from 39 quasiexperimental and six randomized trials indicated that bundles that included audit and feedback were associated with an $82 \%$ increase in hand hygiene [40]. Gould and colleagues [4] found that all 26 studies that met the criteria for inclusion reported some improvement in hand hygiene compliance; however, they were unable to draw conclusions about which interventions or combination of interventions led to clinically important improvements in compliance.

Being tasked with contradictory roles of both "championing" and surveillance audits can be problematic. In real-world settings, fidelity to implementation strategies is often not monitored or measured. As the field of implementation science matures, it is critical to examine how implementation strategies are being used and defined in real-world settings. In addition, implementation science may need to provide guidance for these instances when multiple implementation strategies are being combined or strategies expand beyond the definitional boundaries employed in the implementation science literature. While utilizing champions is an effective approach to eliciting change [31, 32, 42, 43], what guidance can implementation science offer to facilities using $\mathrm{HH}$ champions as a strategy in the real world? In addition, other implementation strategies should be explored to promote hand hygiene compliance.

Qualitative research in infection prevention has increased significantly over the past decade, including in the area of hand hygiene [44-46]. The number of studies using ethnographic methods to examine infection 
prevention interventions has increased since DixonWoods and colleagues examined the Michigan Keystone Project [47] and its attempted replication in England $[19,48]$. One of the strengths of ethnographic and qualitative research is its inductive nature in which engagement with the key individuals and the context can lead to new or more in-depth knowledge and understanding of an area. The ethnographic work being done in infection control and reviewed by Knobloch and colleagues provides evidence of the diversity of deep knowledge that has emerged and strengthened the field from the unintended consequence of mandatory reporting [49] to social and organizational issues that impact hospitalacquired infection rates such as staff shortages and overcrowding [50]. However, few ethnographic studies have focused specifically on hand hygiene [51]. In a very creative and powerful use of ethnographic methods, Hor and colleagues [51] used the method of video reflexive ethnography as a quality improvement intervention in addition to semi-structured interviews and field observations. More broadly, Smiddy and colleagues [46] conducted a systematic qualitative literature review of hand hygiene compliance and found themes could be categorized into two factors: motivational and perceptions of the work environment. These two categories map on to areas often cited as the strengths of ethnographic or qualitative work: stakeholder meaning and structural context [52]. Gilbert and Kerridge [53] is one example in the field of hand hygiene that richly illuminates the welldocumented pattern of physicians being among the least compliant hand washers among healthcare workers. Their in-depth qualitative work in a large, tertiary Australia hospital points to deep cultural, political, and ethical meaning and belief systems that may be contributing to seemingly incongruent pattern of lower compliance, while studies in developing countries point to broader structural and cultural influences impacting hand hygiene compliance among staff within the healthcare settings $[54,55]$. In our own work, we conducted an ethnographic study to examine the everyday practices of hand hygiene programs at 10 different hospitals and found a pattern of a commonly employed implementation strategy: clinical champions. This finding not only has potentially important implications for the hand hygiene field, but also the field of implementation science. This sample of ethnographic and qualitative work focused on hand hygiene highlights points to the need for additional work in this area to examine additional deeply rooted cultural belief systems that may be contributing to compliance or methods of surveillance, as well as the structural and contextual barriers to compliance.

This study is not without limitations. First, our study is restricted to individuals within the VA Healthcare System. Other healthcare systems may use $\mathrm{HH}$ champions differently. Second, our sample was restricted to infection control teams and other individuals involved with hand hygiene and frontline staff available on days the study team visited each facility. Further, phone interviews were limited to only key staff involved with hand hygiene who agreed to participate, and therefore may not be entirely representative of all VA employees. Findings are based on self-report. Although we did observe hand hygiene program practices among some infection preventionists, we did not observe unit level $\mathrm{HH}$ champions as this thematic content emerged from the data and was not a specific element of original data collection. Further ethnographic study of HH champions-including observations of their activities-could serve as the next steps to elucidating the real-world definition and application of the implementation strategy in healthcare contexts. Finally, having an interdisciplinary research team provided the opportunity to challenge the biases we each brought to data collection and analyses. We tracked these conversations in team meetings and in the memo function of MAXQDA software. However, as with all reflexive processes, we may have missed biases that contributed to the interpretation of the results.

\section{Conclusions}

The primary findings of this ethnographic study are twofold. First, it demonstrates how the definition of an implementation strategy (i.e., champion) can move beyond the original definition and encompass elements of additional implementations strategies (e.g., education and training, audit and feedback). This poses an important question to the field of implementation science: how do we understand the effectiveness of strategies when they are combined in ways that have not been evaluated? Secondly, for the field of infection prevention, healthcare systems should consider whether narrowly defining the role of the $\mathrm{HH}$ champion as a dedicated individual whose mission is to support hand hygiene and overcome resistance within an organization-and differentiate it from the role of a "compliance auditor"-could be more impactful in increasing hand hygiene compliance. Returning to the traditional application of the implementation strategy may lead to overall improvements in hand hygiene and reduction of the transmission of healthcare-acquired infections.

\section{Abbreviations \\ EMS: Environmental Management Service; HAls: Healthcare acquired infections; HCW: Healthcare worker; HH: Hand hygiene; HSR\&D: Health Services Research and Development; MDRO: Multi-drug-resistant organisms; VA: Veteran's Affairs; VAHCSs: Veterans Affairs Healthcare Systems; VHA: Veterans Health Administration; WHO: World Health Organization}

Acknowledgements

We are grateful to the site investigators and research coordinators who helped to facilitate the study team's visits and the infection prevention teams and frontline staff who shared their perceptions of hand hygiene. 


\section{Authors' contributions}

CCG and HSR were responsible for data collection and had full access to all of the data in the study and take responsibility for the integrity of the data and the accuracy of the data analysis. HSR, ENP, and MWV were responsible for the concept and design. CCG, DL, MS, NC, MVW, EC, and HSR were responsible for the analysis and interpretation of the data. CCG performed statistical analysis. CCG and HSR drafted the manuscript. All authors provided critical revision of the manuscript for important intellectual content. Funding was obtained by HSR, ENP, and MWV. All authors read and approved the final manuscript

\section{Funding}

Funding support was provided by the Veterans Administration 3 Health Services Research and Development's (VA HSR\&D) Collaborative Research to Enhance and Advance Transformation and Excellence program (grant CRE 12-289).

\section{Availability of data and materials}

The datasets generated and/or analyzed during the current study are not publicly available due to participant privacy but are available from the corresponding author on reasonable request.

\section{Ethics approval and consent to participate}

The study was approved by the VA Central Institutional Review Board and Research and Development Committee at the lowa City VA Health Care System.

\section{Consent for publication}

Not applicable.

\section{Competing interests}

The authors declare that they have no competing interests.

\section{Author details}

'Center for Access \& Delivery Research and Evaluation, lowa City VA Health Care System, 601 Hwy 6 West, lowa City 52246, USA. ${ }^{2}$ Department of Internal Medicine, Carver College of Medicine, University of lowa, 375 Newton Rd, lowa City, IA 52242, USA. ${ }^{3}$ Department of Sociology, University of lowa, 140 Seashore Hall West, lowa City, IA 52242, USA. ${ }^{4}$ Department of Psychological and Brain Sciences, University of lowa, W311 Seashore Hall, lowa City, IA 52242-1407, USA. ${ }^{5}$ University of Utah School of Medicine, 30 N 1900 E, Salt Lake City, UT 84132, USA.

Received: 30 January 2019 Accepted: 24 September 2019 Published online: 23 December 2019

\section{References}

1. Boyce JM, Pittet D. Guideline for hand hygiene in health-care settings. Recommendations of the Healthcare Infection Control Practices Advisory Committee and the HICPAC/SHEA/APIC/IDSA Hand Hygiene Task Force. Society for Healthcare Epidemiology of America/Association for Professionals in Infection Control/Infectious Diseases Society of America. MMWR Recomm Rep. 2002;51(RR-16):1-45 quiz CE41-44.

2. World Health Organization. WHO guidelines on hand hygiene in health care: first global patient safety challenge, clean care is safer care. Geneva: World Health Organization; 2009. http://apps.who.int/iris/bitstream/1 0665/44102/1/9789241597906_eng.pdf. Accessed 20 Oct 2017

3. Kim PW, Roghmann MC, Perencevich EN, Harris AD. Rates of hand disinfection associated with glove use, patient isolation, and changes between exposure to various body sites. Am J Infect Control. 2003;31(2):97-103.

4. Gould DJ, Moraeljo D, Drey N, Chudleigh JH, Taljaard M. Interventions to improve hand hygiene compliance in patient care (review). Cochrane Database Syst Rev. 2017;9:CD005186.

5. Boyce JM, Pittet D, Healthcare Infection Control Practices Advisory Committee. Society for Healthcare Epidemiology of America. Association for Professionals in Infection Control. Infectious Diseases Society of America. Hand Hygiene Task Force. Guideline for hand hygiene in health-care settings: recommendations of the Healthcare Infection Control Practices Advisory Committee and the HICPAC/SHEA/APIC/IDSA Hand Hygiene Task Force. Infect Control Hosp Epidemiol. 2002;23(Suppl12):S3-S40.
6. Pittet D, Allegranzi B, Boyce J, World Health Organization World Alliance for Patient Safety First Global Patient Safety Challenge Core Group of Experts. The World Health Organization guidelines on hand hygiene in health care and their consensus recommendations. Infect Control Hosp Epidemiol. 2009:30(7):611-22.

7. The Joint Commission. National patient safety goals 2018. https://www. jointcommission.org/standards_information/npsgs.aspx. Accessed 20 Oct 2017.

8. Shortell SM, Marsteller JA, Lin M, Pearson ML, Wu SY, Mendel P, Cretin S, Rosen $\mathrm{M}$. The role of perceived team effectiveness in improving chronic illness care. Med Care. 2004;42:1040-8.

9. Powell BJ, Thomas JW, Chinman MJ, Damschroder L, Smith JL, Matthieu MM, Proctor EK, Kirchner JE. A refined compilation of implementation strategies: results from the expert recommendations for implementing change (ERIC) project. Implement Sci. 2015;10:213-4.

10. Beath CM. Supporting the information technology champion. MIS Q. 1991; 15:355-72.

11. Meyer AD, Goes JB. Organizational assimilation of innovations: a multilevel contextual analysis. Acad Manag J. 1998;31(4):897.

12. Schon DA. Champions for radical new inventions. Harv Bus Rev. 1963;41:77.

13. Locock L, Dopson S, Chambers D, Gabbay J. Understanding the role of opinion leaders in improving clinical effectiveness. Soc Sci Med. 2001;53:1.

14. Greenhalgh T, Robert G, Bate P, MacFarlane F, Kyriakidou O. Diffusion of innovations in health service organizations: a systematic literature review. Malden, MA: Blackwell; 2005.

15. White CM, Statile AM, Conway PH, Schoettker PJ, Solan LG, Unaka NI, Vidwan N, Warrick SD, Yau C, Connelly BL. Utilizing improvement science methods to improve physician compliance with proper hand hygiene. Pediatrics. 2012;129(4):e1042-50.

16. Saint S, Conti A, Bartoloni A, Virgili G, Mannelli F, Fumagalli S, Di Martino P, Conti AA, Kaufman SR, MAM R, et al. Improving healthcare worker hand hygiene adherence before patient contact: a before-and-after five-unit multimodal intervention in Tuscany. (Report) Qual Saf Health Care. 2009; 18(6):429 (5).

17. Patel B, Engelbrecht $H$, McDonald H, Morris V, Smythe W. A multifaceted hospital-wide intervention increases hand hygiene compliance. SAMJ. 2016; 106(4):335-41.

18. Cupit C, Mackintosh N, Armstrong N. Using ethnography to study improving healthcare: reflections on the 'ethnographic' label. BMJ Qual Saf. 2018;27(4):258-60.

19. Knobloch MJ, Thomas KV, Patterson E, Zimbic ML, Musuuza J, Safdar N. Implementation in the midst of complexity: using ethnography to study health care-associated infection prevention and control. Am J Infect Control. 2017;45(10):1058-63.

20. Dixon-Woods $\mathrm{M}$. What can ethnography do for quality and safety in health care? Qual Saf Health Care. 2003;12:326-7.

21. Vander Weg M, et al. Does changing point-of-use reminder signs more frequently improve hand hygiene compliance? A cluster randomized trial. JAMA Open. 2019;2(10):e1913823 (in press).

22. Waring JJL. Maintaining the link between methodology and method in ethnographic health research. BMJ Qual Saf. 2016;25(7):554-5.

23. Schensul SL, Schensul JJ, LeCompte MD. Essential ethnographic methods: observations, interviews, and questionnaires. Walnut Creek, CA: AltaMira Press; 1999.

24. Finley EP, Huynh AK, Farmer MM, et al. Periodic reflections: a method of guided discussions for documenting implementation phenomena. BMC Med Res Methodol. 2018;18(1):153.

25. VERBI Software. MAXQDA 2020 [computer software]. Berlin: VERBI Software; 2019. Available from maxqda.commaxqda.commaxqda.com.

26. Agar MH. The professional stranger: an informational introduction to ethnography. 2nd ed. Bingley: Emerald Group Publishing; 1996.

27. Pope CMN. Qualitative research in health care, third edition. Malden: Blackwell Publishing Ltd; 2006.

28. Ryan GW, Bernard RH. Techniques to Identify Themes. Field Methods. 2003; 15:1:85-109.

29. Reisinger HS, Yin J, Radonovich L, Knighton VT, Martinello RA, Hodgson MJ, Perencevich E. Comprehensive survey of hand hygiene measurement and improvement practices in the Veterans Health Administration. Am J Infect Control. 2013;1-5.

30. Chen LF, Vander Weg MW, Hoffman DA, Reisinger HS. The Hawthorne Effect in Infection Prevention and Epidemiology. Infect Control Hosp Epidemiol. 2015;36(12):1444-50. 
31. Damschroder LJ, Banaszak-Holl J, Kowalski CP, Forman J, Saint S, Krein SL. The role of the "champion" in infection prevention: results from a multisite qualitative study. Qual Saf Health Care. 2009;18:434-40.

32. Soo S, Bereta W, Baker, RG. Role of champions in the implementation of patient safety practice change. Healthc Q. 2009;12 Special Issue:123-8.

33. Hendy J, Barlow J. The role of the organizational champion in achieving health system change. Soc Sci Med. 2012;74:348-55.

34. McInnes E, Phillips R, Middleton S, Gould D. A qualitative study of senior hospital managers' views on current and innovative strategies to improve hand hygiene. BMC Infect Dis. 2014;14:611.

35. Miech EJ, Rattray NA, Flanagan ME, Damschroder L, Schmid AA, Damush T. Inside help: an integrative review of champions in healthcare-related implementation. SAGE Open Med. 2018;6:1-11.

36. Kitson AL, Rycroft-Malone J, Harvey G, McCormack B, Seers K, Titchen A. "Evaluating the successful implementation of evidence into practice using the PARiHS framework: theoretical and practical challenges." Implement Sci. 2008:3:1.

37. Harvey G, Kitson A. PARIHS revisited: from heuristic to integrated framework for the successful implementation of knowledge into practice. Implement Sci. 2016;10:33.

38. Harvey G, Kitson A. Translating evidence into healthcare policy and practice: single versus multi-faceted implementation strategies-is there a simple answer to a complex question? International journal of health policy and management. 2015;4(3):123.

39. Ivers N, Jamtvedt G, Flottorp S, Young JM, Odgaard-Jensen J, French SD O'Brien MA, Johansen M, Grimshaw J, Oxman AD. Audit and feedback: effects on professional practice and healthcare outcomes. Cochrane Database Syst Rev. 2012;6.

40. Livorsi DJ, Goedken CC, Sauder M, VanderWeg MW, Perencevich EN, Reisinger, HS. Evaluation of barriers to audit-and-feedback programs that used direct observation of hand hygiene compliance a qualitative study. JAMA Open. 2018;1(6):1-11.

41. Schweizer ML, Reisinger HS, Ohl M, Formanek MB, Blevins A, Ward MA, Perencevich EN. Searching for an optimal hand hygiene bundle: a metaanalysis. Clin Infect Dis. 2014;58(2):248-59.

42. Kirchner J, Parker L, Bonner L, Fickel J, Yano E, Ritchie M. Roles of managers, frontline staff and local champions, in implementing quality improvement: stakeholders' perspectives. J Eval Clin Pract. 2012;18:63-69.

43. Ploeg J, Skelly J, Rowan M, Edwards N, Davies B, Grinspun D, Bajnok I, Downey $\mathrm{A}$. The role of nursing best practice champions in diffusing practice guidelines: a mixed methods study. Worldviews Evid Based Nurs. 2010; Fourth Quarter:238-251.

44. Krein, SL, Olmsted, RN, Hofer, TP, Kowalski, C, Forman, J, Banaszak-Holl, J, Saint, S. Translating infection prevention evidence into practice using quantitative and qualitative research. Am J Infect Control. 2006;34(8): 507-12.

45. Forman, J, Creswell, JW, Damschroder, L, Kowalski, CP, Krein, SL. Qualitative research methods: key features and insights gained from use in infection prevention research. Am J Infect Control. 2008;36(10):764-71.

46. Smiddy, MP, O'Connell, R. Creedon, SA. Systematic qualitative literature review of health care workers' compliance with hand hygiene guidelines. Am J Infect Control. 2015;43(3):269-74.

47. Dixon-Woods M, Bosk CL, Aveling EL, Goeschel CA, Pronovost PJ. Explaining Michigan: Developing an Ex Post Theory of a Quality Improvement Program. Milbank Quarterly. 2011;89(2):167-205.

48. Dixon-Woods M, Leslie M, Bion J, Tarrant C. What counts? An ethnographic study of infection data reported to a patient safety program. Milbank Quarterly. 2012;90(3):548-91.

49. Szymczak J.E. Seeing risk and allocating responsibility: talk of culture and its consequences on the work of patient safety. Soc Sci Med. 2014;120:25225950.

50. VanSteelandt, J. Conly, W. Ghali, C. Mather. Implications of design on infection prevention and control practice in a novel hospital unit: the medical ward of the 21st century. Anthropol Med. 2015;22:149-16151.

51. Hor, S-y, Hooker, C, ledema, R, Wyer, M, Gilbert, GL, Jorm, C, O'Sullivan, MVN. Beyond hand hygiene: a qualitative study of the everyday work of preventing cross-contamination on hospital wards. BJM Qual Saf. 2017;26: 552-8.

52. Sobo, EJ. Culture and meaning in health services research: an applied approach. First Edition. New York: Left Coast Press, Inc.; 2009.
53. Gilbert, GL, \& Kerridge, I. The politics and ethics of hospital infection prevention and control: a qualitative case study of senior clinicians' perceptions of professional and cultural factors that influence doctors' attitudes and practices in a large Australian hospital. BMC Health Serv Res. 2019;2;19(1):212

54. Marjadi, B \& McLaws, ML. Hand hygiene in rural Indonesian healthcare workers: barriers beyond sinks, hand rubs and in-service training. J Hosp Infect. 2010;76(3):256-60.

55. González, ML, Finerman, R, Johnson, KM, Melgar, M, Somarriba, MM, Antillon-Klussmann, F, Caniza, MA. Understanding hand hygiene behavior in a pediatric oncology unit in a low- to mid-income country. J Nurs Educ Pract. 2016;6(9):1-9.

\section{Publisher's Note}

Springer Nature remains neutral with regard to jurisdictional claims in published maps and institutional affiliations.

\section{Ready to submit your research? Choose BMC and benefit from:}

- fast, convenient online submission

- thorough peer review by experienced researchers in your field

- rapid publication on acceptance

- support for research data, including large and complex data types

- gold Open Access which fosters wider collaboration and increased citations

- maximum visibility for your research: over $100 \mathrm{M}$ website views per year

At BMC, research is always in progress.

Learn more biomedcentral.com/submissions 